\title{
A Model for Improved Estimates of Child Wasting in Yemen: Capturing the Effects of Conflict and Seasonal Variability In Incidence Estimates
}

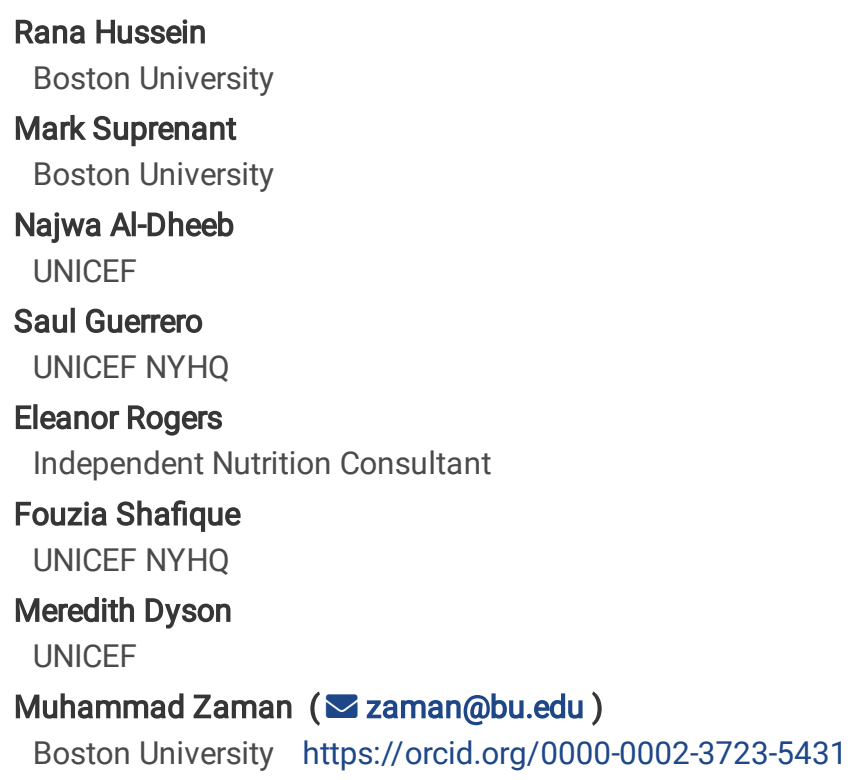

Research

Keywords: Yemen, acute malnutrition, wasting, mathematical modeling, incidence correction factor

Posted Date: November 20th, 2020

DOI: https://doi.org/10.21203/rs.3.rs-110943/v1

License: @ (i) This work is licensed under a Creative Commons Attribution 4.0 International License. Read Full License 


\section{Abstract}

Introduction: Conflict-affected settings - where starvation is often used as a weapon of war and deliveries of food restricted by warring parties, millions are displaced and economies are crippled, and health infrastructure destroyed - have become increasingly linked to high rates of wasting in children. In the case of Yemen, this relationship has become strikingly clear. The country's ongoing civil war has severely restricted imports of food and disrupted livelihoods, worsening already high pre-war levels of food insecurity. Paired with outbreaks of disease and an overwhelmed and underfunded health system, this has brought rates of wasting in children under five - who are particularly vulnerable to and disproportionately impacted by wasting - to unprecedented levels, which continue to increase as the crisis worsens and aid becomes increasingly limited. In their planning of services to treat and prevent moderate and severe wasting in children, humanitarian agencies estimate expected caseload using a single, standard estimate. This calculation is inapplicable to the context of Yemen both because it is based on a global estimate and rates of incidence vary by context and because it does not capture variations in incidence due to seasonality, disease trends, and the general instability of a crisis setting. To address these limitations and provide more holistic and context-specific estimates of the incidence of wasting among children in Yemen, we developed a predictive Markov model. Computing context-specific values for the average duration of an episode of wasting, the model then considers how changes to other factors in Yemen - food insecurity levels and seasonal disease trends - cause incidence to vary.

Methods: We developed a Markov model to estimate the monthly incidence and resultingly, prevalence, of moderate and severe wasting among under-five children in the governorate of Lahj, Yemen. Transition rates were estimated using a combination of monthly treatment analysis compiled by the Yemen Nutrition Cluster and provided by UNICEF, annual SMART survey prevalence estimates and other estimates from the literature. Through model calibration, context-specific values for the average duration of an episode of moderate and severe wasting, and respective incidence correction factors, were found. Local food insecurity levels and diarrheal disease rates - factors directly associated with the incidence of wasting - were introduced as adjustable parameters that would affect monthly incidence rates based on established mathematical relationships.

Results: The calculated context-specific incidence correction factor for Yemen showed that previous estimates led to considerable underestimates of the burden of wasting. Adjusted annual caseloads for moderate and severe wasting were $29 \%$ and $40 \%$ higher, respectively, than the previously assumed values. Baseline values for the estimated duration of an episode of moderate and severe wasting were found to be 5.3 and 4.8 months, respectively. When these values were taken as the average duration of an episode of wasting and the model was run from September 2018 to October 2019, the model's resulting estimates of the prevalence of moderate and severe wasting matched those recorded in the October 2019 SMART survey. By accounting for changes to underlying factors of wasting, the model produced outputs that reflect the variability of monthly incidence rates of wasting, mirroring the fluctuations seen in treatment admissions data.

Conclusion: In this manuscript we propose a Markov model for more accurate and holistic estimates of the burden of wasting in children under five. By generating context specific incidence rates based on levels of food insecurity and seasonal disease trends, our estimates for the duration of an episode and thus caseload more accurately capture changing realities on the ground in Yemen. While we applied our model to Yemen, this model is highly flexible and may be used in other conflict-affected settings to allow health care workers to better predict and plan for expected cases of wasting.

\section{Introduction}

Food security crises are closely linked to armed conflict [1]. As a result of both the widespread destruction and devastation that violent conflict causes as well as many of the indirect effects of war which disrupt the daily lives of civilians, conflict creates many of the conditions that drive hunger. Widespread food insecurity leaves young children among the most vulnerable to wasting, a rapid deterioration in nutritional status over a short period of time; children often suffer more severe and irreversible consequences due to wasting[2]. It is estimated that 112 million wasted children live in conflict-affected areas [3]. As a result of conflict, millions of civilians are displaced and left particularly vulnerable to wasting and cut off from their sources of food and safe water, livelihoods and social networks. War also frequently disrupts the food supply - with starvation used as a tactic of war and warring parties deliberately restricting the distribution of food and critical supplies or through the destruction of farms and livestock. In contexts where medical services are also extremely limited and much of the health infrastructure destroyed, conflict-affected areas often see high rates of untreated disease, exacerbating the risk of wasting in children in particular.

In Yemen, where the country's current hunger crisis is largely the result of warring parties' deliberate efforts to restrict food, and the war has crippled the economy and disrupted livelihoods, the role of conflict in creating the conditions that create widespread wasting among children is strikingly clear. The ongoing civil war in Yemen has resulted in what has been called the worst humanitarian crisis in the world, 
pushing the country to the brink of famine. A blockade on Yemeni ports has severely restricted imports of food, fuel, and critical medical supplies [4]. Severe food shortages, soaring food prices and disrupted livelihoods have made food and basic necessities unaffordable to many and have led to widespread food insecurity, which, paired with outbreaks of disease and an overwhelmed and chronically underfunded health system, have created unprecedented levels of wasting in children under five [5]. Child wasting rates in Yemen are among the highest in the world and continue to increase as the crisis worsens and aid becomes increasingly limited; in some areas of Yemen, it is estimated that more than one in four children suffer from wasting [5]. Untreated wasting can permanently impair a child's cognitive and physical development and places them at an increased risk of morbidity and mortality; a malnourished child is much more vulnerable to more severe disease and recurrent infections [2]. Wasting severely weakens the immune system's capacity to fight infections, making otherwise mild infections fatal for wasted children. Humanitarian agencies are working across Yemen to address a worsening crisis of widespread wasting among children. Nutrition interventions are implemented according to the Community-Based Management of Acute Malnutrition (CMAM) Model, the globally endorsed standard for management of acute malnutrition, also known as wasting, which aims to reduce mortality and morbidity from wasting by providing early case-finding and effective treatment and by strengthening the local community's capacity to prevent, identify and manage wasting.

Since the conflict in Yemen began in 2015, humanitarian agencies have scaled up efforts to both prevent malnutrition and identify and treat cases of Global Acute Malnutrition - Moderate Acute Malnutrition (MAM), also known as moderate wasting, and Severe Acute Malnutrition (SAM), also known as severe wasting- in children under five. (Though the terms wasting and acute malnutrition are often used interchangeably, there exists a subtle difference between them. Acute malnutrition is the umbrella term under which wasting falls; acute malnutrition is defined by the presence of wasting and/or bilateral pitting nutritional oedema [6]. However, the terms moderate and severe wasting will be used throughout this manuscript to refer to the broader category of acute malnutrition; this is in accordance with the recent shift within the public health and nutrition community towards a generalized use of the term wasting to refer to acute malnutrition as defined by Weight-for-height Z-Score (WHZ), mid-upper arm circumference (MUAC) and/or oedema.)

For planning purposes, humanitarian agencies use prevalence estimates and historical program data to estimate the expected number of cases of moderate and severe wasting as well as expected treatment coverage for the coming year. Reliable estimates of caseload are needed for policy decisions and the planning of services and are critical in a context where funding and resources are extremely limited; however, the current, standardly-used approach to estimate needs and plan accordingly presents several major limitations that result in inaccurate estimates and provide little insight that can effectively guide planning decisions.

First, given that context-specific information about incidence rates and outcomes of wasting in Yemen is limited, estimates of annual wasting caseloads rely on a universally-used calculation of incidence proposed for use in the CMAM model [7]. This calculation uses the number of prevalent cases and an estimate of the average duration of an untreated episode of moderate and severe wasting to estimate incidence. The value taken as the average duration of an untreated episode, 7.5 months, was found from two cohort studies conducted in two African nations in the 1980s and is currently used globally [8]. However, recent cohort studies tracking the progression of untreated cases of wasting have shown that this value varies widely by context and in all contexts studied, using this value led to a considerable underestimation of caseload [9]. This is further evidenced by historical program data from Yemen showing that the number of children treated during a given period exceeds the estimated caseload, with reported treatment coverage rates greater than one hundred percent. Additionally, with this value assumed to remain unchanged from year to year, the only source of variance in the current annual caseload calculation comes from cross-sectional prevalence data. Because of this, estimated caseloads for wasting in Yemen over the last three years have remained relatively constant, which does not align with the general instability caused by the conflict or extensive reports showing that the nutrition situation has continued to deteriorate over the past years. Basing all planning decisions on annual estimates of prevalence - a function of incidence, recovery and treatment rates, and fatality rates - provides little insight that can be used to guide policy decisions.

Second, the current approach assumes incidence rates to be constant throughout the year, neglecting known variations in incidence from month to month. In Yemen, as in most contexts, incidence rates are directly affected by changes in other factors including seasonal trends in food insecurity levels as well as disease trends - all highly variable due to the conflict. Without a consideration of these variations, current estimates also do not capture large-scale changes in incidence rates that have occurred since the conflict began, such as the expected increases in rates of wasting when, for example, the conflict severely restricts food imports, or when diarrheal disease outbreak occurs. These estimates also do not capture expected monthly fluctuations throughout the year, an understanding of which provides important insights about how needs will change, which can guide decisions about how resources and services should be allocated most effectively.

Lastly, the current approach to estimate the respective caseloads of moderate wasting, severe wasting with and without complications examines each one independently of the others, neglecting the paths between them and ways in which they inherently interact to form a connected system. For example, when humanitarian agencies scale up their implementation of treatment programs for moderate wasting,

Page 3/19 
this will not only reduce the burden of moderate wasting but will also likely reduce the burden of severe wasting and severe wasting with complications in the long-term. Without capturing the bidirectional paths between the various forms of wasting, the burden of wasting and the respective impacts of various interventions cannot be holistically assessed.

In order to address these limitations and provide more holistic and context-specific estimates of the burden of wasting in Yemen, we developed a predictive Markov model, a model commonly used in epidemiology to model the progression of disease. Our model examines the governorate of Lahj in Yemen. Taking adjusted, context-specific rates of incidence, Yemen Nutrition Cluster data presenting rates and outcomes of various treatments (provided by UNICEF) and information about other parameters including food insecurity levels and seasonal disease trends, our model provides a context-specific and holistic representation of the nutrition situation. It seeks to capture the complete system of interactions between incidence rates, the different stages in the progression of wasting, as well as rates of treatment and their respective outcomes, which collectively determine the burden of wasting, in order to predict the number of under-five children who have or are in treatment for moderate wasting, severe wasting, and severe wasting with complications each month in Lahj.

The model's context-specific incidence rates allow for more accurate moderate and severe wasting caseload estimates and its incorporation of underlying factors as inputs allows for a consideration of how caseloads change as conditions in Yemen change. By incorporating factors known to directly impact the nutrition situation such as food insecurity levels and seasonal disease trends - all highly variable as a result of the conflict - as tunable parameters, the model provides a context-specific representation of ever-changing realities on the ground. Though many of the relationships that the model captures are known among individuals on the ground, (for example, it is known that rates of wasting tend to increase during the rainy season, when diarrheal disease rates are at their highest) these relationships only exist in the form of anecdotal evidence. The model provides a more concrete and systemic understanding of these factors and their respective impacts on wasting rates by capturing how future changes to any of these factors will affect the nutrition situation. Additionally, because the model captures the ways in which moderate wasting, severe wasting and severe wasting with complications all inherently interact, decisionmakers can modify the model's parameters, either to reflect changing realities on the ground or to simulate the consequences of a potential decision, such as prioritizing a given intervention, allowing the model's outputs to guide decisions about future interventions. For example, decision-makers may modify rates of treatment for moderate wasting as an input to the model in order to understand how scaling up interventions for moderate wasting will also impact the burden of severe wasting and severe wasting with complications in the long term. The model may provide humanitarian agencies with insights about how needs will change over time and allow them to plan accordingly and allocate already limited funding and resources most effectively.

\section{Methods}

The burden of wasting is determined by a set of paths between moderate wasting, severe wasting and severe wasting with complications as well as treatment admissions and outcomes, creating a network of states for children to move between. This system easily maps onto the general framework of a Markov model. Given this, a Markov model was used to represent this system and provide usable outputs to health workers in the field. These models are commonly used for probabilistic modeling, especially in fields like epidemiology where there are a defined number of outcomes or states being modeled [10]. A child can be classified according to their nutritional status as being either non-malnourished, malnourished but not in treatment, in-treatment for wasting or deceased. The Markov model presented in this work distinguishes between each level of wasting (moderate wasting, severe wasting and severe wasting with complications) and its respective treatment program. It is composed of eight nutritional statuses: Healthy, Moderately Wasted, Severely Wasted, Severely Wasted with Complications, Treatment $_{M}$, Treatment ${ }_{S}$ Treatment $_{S C}$ and Deceased. If a child resides in either the Moderately Wasted, Severely Wasted, or Severely Wasted with complications state, they are malnourished but not currently in treatment. However, because the model does account for rates of defaulters (children who stop attending treatment appointments before reaching discharge criteria) and non-responders (children who fail to respond to treatment), if a child currently resides in any of these three states, this does not necessarily mean they have never been admitted to treatment and hence these states are not referred to as untreated. During the model's simulation, children can move between states at a set of monthly transition rates, representing the probability that a child in one state will move to another (or remain in the same one) from one month to the next. All possible transitions are depicted by the arrows in Figure 1. By knowing the number of children initially residing in each state, the model can be run for any number of months and produce a monthly breakdown of the expected number of children in each nutritional state.

The model is based on the following assumptions:

1. A child must reside in a malnourished state (Moderately Wasted, Severely Wasted or Severely Wasted with complications) before entering a treatment state. 
2. A child cannot transition from the Healthy state directly to the Severely Wasted state; a child residing in the Severely Wasted state must have previously resided in the Moderately Wasted state.

3. It is assumed that spontaneous recovery can occur among cases of moderate wasting.

4. A severely wasted child will not return to the Healthy state without first residing in either the state or the Moderately Wasted state. A child cannot transition directly from the Severely Wasted state to the Healthy state; however, they may transition to Moderately Wasted and from Moderately Wasted, return to the Healthy state.

5. A child in treatment for any form of wasting may default from or fail to respond to treatment, in which case they would transition from the treatment state back to the malnourished state (Moderately Wasted, Severely Wasted, or Severely Wasted with complications) in which they previously resided.

6. The Deceased state is an absorbing state.

\section{Determining the Initial State Distribution}

Given that it is known that at any point in time, a certain population of under-five children in Lahj is either suffering from or in treatment for wasting and that children are expected to remain in either the wasted or treatment state for several months, it would be unreasonable to begin the model's simulation with no cases of wasting both because this does not reflect reality and because all subsequent rate calculations would be skewed. The initial state distribution, describing the number of children starting in each nutritional state, aimed to reflect this. The model's initial state distribution was determined by 2018 cross-sectional SMART survey data available at the Nutrition Cluster level, presenting the prevalence of moderate and severe wasting among under-five children in the Lahj governorate using the WeightFor-Height Z-Score as the screening metric [11]. Using district-level estimates of the under-five population of Lahj and SMART district-level estimates of the prevalence of moderate and severe wasting, the number of children estimated to be moderately or severely wasted at the time of the survey was calculated. Data from the Yemen Nutrition Cluster was used to estimate the average number of children enrolled in moderate wasting treatment (Targeted Supplementary Feeding Programs) and severe wasting treatment (Outpatient Therapeutic Feeding Programs) each month. Because children enrolled in treatment will remain in treatment for more than one month, it was important for the model to capture the fact that during any given month, among the children currently enrolled in treatment programs, (those currently residing in the treatment state) only some of them will have been admitted in the current simulated month and only some of them will be discharged in the current simulated month. These considerations guided the calculation of all transition rates for entering or leaving treatment, discussed in the following section in greater detail.

\begin{tabular}{|c|c|}
\hline Nutritional State & Number of Children \\
\hline Healthy & 153612 \\
Severely Wasted & 2896 \\
Moderately Wasted & 17179 \\
Severely Wasted with complications & 0 \\
& 8494 \\
& 3219 \\
Deceased & 0 \\
\hline
\end{tabular}

Table 1. Model's Initial State Distribution.

While the SMART procedure does not distinguish between untreated wasted children and those who are enrolled in treatment programs, our model made this distinction by designating two different states for treated and untreated children, which required that several simplifying assumptions be made. For severe wasting, it was assumed that among those enrolled in treatment, only those admitted within the past month would still satisfy the Z-Score used to classify severe wasting cases (Z-Score <-3.0) and were therefore included in the estimated number of severe wasting cases produced by SMART [6]. The remaining cases of severe wasting were assumed to be untreated and would be found in the Severely Wasted state. The same assumptions were made about cases of moderate wasting $(-3.0<$ Z-Score $<-2.0)$. Given that severe wasting with complications is associated with a high risk of mortality and children are unlikely to remain with complicated severe wasting for several months, it was assumed that there would be no cases of complicated severe wasting in the initial distribution. Because in-patient treatment for complicated severe wasting generally takes less than one month, it was assumed that no children were in treatment for severe wasting with complications to start. The initial state distribution is shown in Table 1. Given that the prevalence of wasting varies considerably by season, because the SMART survey used for these estimations was conducted in September, it was 
established that the model would begin its simulated year in September. The model would be run until October 2019 - the date of the next SMART survey - in order to provide a basis for comparison for the resulting prevalence of moderate and severe wasting [12].

\begin{tabular}{|c|c|c|}
\hline Nutrition State Transition & Interpretation & $\begin{array}{c}\text { Transition } \\
\text { Rate }\end{array}$ \\
\hline \multicolumn{3}{|l|}{ From Healthy State } \\
\hline Healthy to Healthy $\left(p_{\text {HH }}\right)$ & Remains healthy & $\begin{array}{r}1-p_{H M}- \\
0.00966\end{array}$ \\
\hline Healthy to Moderately Wasted $\left(p_{H M}\right)$ & $\begin{array}{l}\text { Incidence of Moderate } \\
\text { Wasting }\end{array}$ & Equation \\
\hline Healthy to Severely Wasted ( $\left.p_{H S}\right)$ & $\begin{array}{c}\text { Develops severe wasting } \\
\text { from healthy }\end{array}$ & 0 \\
\hline Healthy to Severly Wasted with complications $\left(p_{H C}\right)$ & $\begin{array}{c}\text { Develops severe wasting } \\
\text { w/ complication from } \\
\text { healthy }\end{array}$ & 0 \\
\hline Healthy to Deceased $\left(p_{H D}\right)$ & $\begin{array}{l}\text { General Under-five } \\
\text { Mortality Rate }\end{array}$ & 0.00966 \\
\hline \multicolumn{3}{|l|}{ From Severelly Wasted State } \\
\hline Severely Wasted to Healthy $\left(p_{S H}\right)$ & $\begin{array}{c}\text { Severe Wasting } \\
\text { Spontaneous Recovery }\end{array}$ & 0 \\
\hline Severely Wasted to Moderately Wasted $\left(p_{S M}\right)$ & $\begin{array}{l}\text { Untreated severe wasting } \\
\text { improves to moderate } \\
\text { wasting }\end{array}$ & 0.0559 \\
\hline Severely Wasted to Severely Wasted $\left(p_{s s}\right)$ & $\begin{array}{l}\text { Severe wasting remains } \\
\text { severe wasting }\end{array}$ & 0.682 \\
\hline Severly Wasted to Severely Wasted with complications ( $p_{s c}$ ) & $\begin{array}{c}\text { Untreated severe wasting } \\
\text { develops medical } \\
\text { complication }\end{array}$ & 0.0352 \\
\hline Severely Wasted to Treatments $\left(p_{S T_{T}}\right)$ & Admitted to OTP & 0.128 \\
\hline Severely Wasted to Deceased $\left(p_{S D}\right)$ & $\begin{array}{c}\text { Untreated Severe Wasting } \\
\text { Case Fatality }\end{array}$ & 0.0989 \\
\hline $\begin{array}{l}\text { From Moderately Wasted State } \\
\text { Moderately Wasted to Healthy }\left(p_{M H}\right)\end{array}$ & $\begin{array}{l}\text { Moderate Wasting } \\
\text { Spontaneous Recovery }\end{array}$ & 0.0275 \\
\hline Moderately Wasted to Severely Wasted ( $\left.p_{M S}\right)$ & $\begin{array}{l}\text { Untreated moderate } \\
\text { wasting progresses to } \\
\text { severe wasting } \\
\text { (severe wasting incidence) }\end{array}$ & Equation \\
\hline Moderately Wasted to Moderately Wasted ( $\left.p_{M M}\right)$ & $\begin{array}{l}\text { Moderate wasting remains } \\
\text { moderate wasting }\end{array}$ & $\begin{array}{c}1-p_{Q S 5}- \\
0.165\end{array}$ \\
\hline Moderately Wasted to Treatment $t_{M}\left(p_{M T_{M}}\right)$ & Admitted to TSFP & 0.157 \\
\hline Moderately Wasted to Deceased ( $\left(p_{M D}\right)$ & $\begin{array}{l}\text { Untreated Moderate } \\
\text { Wasting Case Fatality }\end{array}$ & 0.00357 \\
\hline $\begin{array}{l}\text { From Severelv Wasted with complications State } \\
\text { Severely Wasted with complications to Severely Wasted with complications (pcc) }\end{array}$ & $\begin{array}{l}\text { Severely wasted } \mathrm{w} / \\
\text { complications remains } \\
\text { severely wasted w/ } \\
\text { complications }\end{array}$ & 0.2996 \\
\hline Severely Wasted with complications to Treotment $s_{C}\left(p_{C_{T} T_{0}}\right)$ & Admitted to TFC & 0.1821 \\
\hline Severely Wasted with complications to Deceased $\left(p_{C D}\right)$ & $\begin{array}{c}\text { Severely Wasted w/ } \\
\text { complications Untreated } \\
\text { Case Fatality }\end{array}$ & 0.5183 \\
\hline \multicolumn{3}{|l|}{ From Ireatments State } \\
\hline Treatments to Severely Wasted ( $\left.p_{T, s}\right)$ & Defaults from OTP & 0.169 \\
\hline Treatments to Treatment $H_{M}\left(p_{T T_{W}}\right)$ & Referred from OTP to TSFP & 0.00234 \\
\hline Treatments to Treotments $\left(p_{T, \tau_{s}}\right)$ & Remains in OTP & 0.606 \\
\hline Treatments to TreatmentsC $\left(p_{T T S}\right)$ & Referred from OTP to TFC & 0.00307 \\
\hline Treatments to Deceased $\left(p_{T_{s}} D\right)$ & $\begin{array}{c}\text { In-treatment (OTP) case } \\
\text { fatality }\end{array}$ & 0.00329 \\
\hline \multicolumn{3}{|l|}{ From Ireatmentu State } \\
\hline Treatment $t_{M}$ to Moderately Wasted $\left(p_{T_{M} M}\right)$ & Default from TSFP & 0.20060 \\
\hline Treatment $_{M}$ to Treatment $t_{M}\left(p_{T_{M} T_{m}}\right)$ & Remains in TFSP & 0.735 \\
\hline Treatment $t_{M}$ to Treatments $\left(p_{T_{T}, T_{S}}\right)$ & Referred to OTP from TSFP & 0.0548 \\
\hline Treatment $t_{M}$ to Deceased $\left(p_{T_{m} D} D\right)$ & $\begin{array}{c}\text { In-treatment (TSFP) case } \\
\text { fatality }\end{array}$ & 0 \\
\hline \multicolumn{3}{|l|}{ From Ireatmentsc State } \\
\hline Treatmentsc to Healthy $\left(p_{T_{C} H}\right)$ & Cured at TSFP & 0.728 \\
\hline 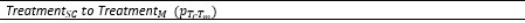 & Referred from TFC to TSFP & 0.0784 \\
\hline Treatments to treatments $\left(p_{T_{C} T_{S}}\right)$ & Referred from TFC to OTP & 0 \\
\hline Treatments to treatmentsc $\left(p_{T_{T}, T_{c}}\right)$ & Remains in TFC & 0.0540 \\
\hline Treatmentsc to Deceased $\left(p_{T_{T}, D}\right)$ & $\begin{array}{l}\text { In-treatment (TFC) case } \\
\text { fatality }\end{array}$ & 0.140 \\
\hline $\begin{array}{l}\text { From Deceased State } \\
\text { Deceased to Deceased }\left(p_{D D}\right)\end{array}$ & $\begin{array}{l}\text { Deceased state is } \\
\text { absorbing }\end{array}$ & 1 \\
\hline
\end{tabular}

Table 2. List of Model's Transition Rates. Each transition rate represents the probability that a child in a given nutritional state moves to another within one month. All unlisted rates have a value of 0 .

Determining_Model's Fixed Rates: Treatment Outcomes, General Mortality, Spontaneous Recovery, Untreated Case Fatality.

Though many of the model's rates aimed to reflect their expected variability throughout the year and were therefore time-varying, several of the model's rates were assumed to be constant. Treatment outcome rates were estimated from CMAM compiled data provided by the UNICEF Yemen country office which followed children enrolled in Targeted Supplementary Feeding Programs (TSFPs) for moderating wasting treatment, Outpatient Therapeutic Programs (OTPs) for severe wasting treatment and Therapeutic Feeding Centers (TFCs) for complicated severe wasting treatment. (This data is available at the Yemen Nutrition Cluster included information regarding all CMAM nutritional interventions implemented in the governorate.) Transition rates from any treatment state back to the non-malnourished state was estimated from their respective program cure rates. Rates of transfer between various treatment programs (OTP, TSFP, and TFC) were also accounted for as shown in Table 2. Transition rates from any treatment state back to a wasted state were estimated using rates of defaulters and non-responders.

The general under-five mortality rate estimated by El Bcheraoui et. al - using estimates from the governorate-level estimates from the 2013 Yemen National Health and Demographic Survey (DHS) and supplementing them with estimates of mortality due to the conflict - was used to estimate the model's general mortality rate [13]. In the absence of spontaneous recovery estimates specific to Yemen - and due to a general scarcity of reporting of spontaneous recovery of wasting - rates of spontaneous recovery for moderate wasting were estimated using data from a cohort study following moderately wasted under-five children in Iran [14]. Though the contexts of Iran and Yemen differ in several ways, this estimate was taken among the limited available estimates as the most comparable to Yemen. The implications of doing 
so ar4e discussed in greater detail later in the manuscript. The rate at which an untreated severely wasted child improved to become moderately wasted was derived from a cohort study measuring the efficacy of various treatments; this rate was estimated based on rates of reported outcomes of children in the untreated control group [15]. Given the scarcity of estimates of untreated wasting case fatality rates specific to Yemen, the model's untreated moderate and severe wasting case fatality rates were derived from hazard ratios estimated by a pooled meta-analysis using data from cohorts across different contexts before the onset of CMAM [16].

\begin{tabular}{|c|c|c|}
\hline Notation & Value Type & Description \\
\hline FI $(q)$ & Equation & Food Insecurity value for quarter $q$ \\
\hline Qduration $_{\mathrm{q}}$ & Equation & Duration for quarter $q$ after applying FI \\
\hline avgdur $_{M}$ & Constant & Computed baseline average duration of moderate wasting episode in months \\
\hline avgdur $_{S}$ & Constant & Computed baseline average duration of severe wasting episode in months \\
\hline Qincidence $_{q}$ & Equation & Incidence for quarter $q$ after applying FI effect \\
\hline Mincidence & Equation & Incidence for month $m$ calculated by applying quarterly FI effect and monthly rainfall effect \\
\hline rainfall & Monthly Value & Monthly value in range [0, 0.1] based on rainfall and temperature \\
\hline
\end{tabular}

Table 3. Table of values used throughout manuscript and descriptions.

\section{Adjusting Incidence Rates}

Given that a direct estimate of incidence rates of wasting would involve following a cohort of untreated children over an extended period, incidence generally cannot be directly observed. This is especially true in conflict-affected zones such as Yemen, where resources are limited and it would be impractical to conduct frequent surveys to assess the burden of wasting. In the absence of incidence data, incidence can be estimated using a simple mathematical relationship involving the annual prevalence and average duration of an episode, approximating incidence as incidence/average duration of an episode. Global guidelines recommend an estimate of 7.5 months as the average duration of an episode of wasting, yielding an incidence correction factor of 1.6 for a planning period of one year [7]. In almost all contexts studied, this estimate has been shown to yield considerable underestimates of incidence $[17,18,9,19]$. When severe and moderate wasting incidence rates were calculated using this method in order to estimate the model's wasting incidence rates, when run for one year, the model's estimates of the prevalence of moderate and severe wasting the end of the year were lower than those recorded from the following year's SMART survey for Lahj, as shown in Figure 2. This, along with the extensive evidence showing that the estimated 7.5-month duration is inaccurate and cannot be applied to all contexts, indicates that an adjusted, context-specific estimate of incidence is needed [17, $18,9,19]$.

Given this, we aimed to estimate a context-specific incidence rate for both moderate and severe wasting in Yemen that not only reflected what seemed to be a significantly higher rate of incidence than the one estimated using a standard incidence correction factor, but also considered the inherent variability of rates of wasting in a crisis setting.

\section{Finding a Context-specific Estimate for the Average Duration of An Episode of Moderate and Severe Wasting}

Given that the estimated 7.5-month duration of an episode, and its associated incidence correction factor of 1.6, were producing underestimates of wasting caseloads, an adjusted, context-specific estimate was found. We aimed to find an estimate of the average duration of an episode of severe wasting which, when used to run the model for 14 simulated months, would result in a prevalence of severe wasting that matched the value reported in the 2019 SMART survey results [11]. Without yet considering the effects of seasonal variability, this estimate would function as a baseline estimate for the average duration of severe wasting, where it would be assumed that this value remained constant throughout the year. Using the initial state distribution informed by 2018 data shown in Table 1 as well as 
known estimates of each of the model's non-incidence rates, the model was run for fourteen simulated months (until October 2019) calibrated with various different estimates of severe wasting incidence rates, in order to find an estimate which resulted in a severe wasting prevalence that matched that shown in the October 2019 SMART survey for Lahj. (In the process of model calibration, all rates corresponding to treatment admissions were adjusted on a monthly basis in order to match the recorded values from the data given that admissions were not constant throughout.) In our model, children can leave the Severely Wasted state either by entering the state, entering the Severely Wasted with complications state, entering the Moderately Wasted state or entering the Deceased state. Children enter the Severely Wasted state when they develop severe wasting from moderate wasting, representing the rate of incidence. As shown in Figure 3 these six rates collectively determine the prevalence of severe wasting each month. With all but incidence known, incidence could be modulated in order to find an estimate which produces the correct prevalence after running the model for a simulated period from September 2018 to October 2019. This value is referred to as avgdur.

The same process of model calibration was used to estimate the average duration of a moderate wasting episode. However, because the model framework assumes all severe wasting cases develop from cases of moderate wasting, the prevalence of moderate wasting is also directly affected by the incidence of severe wasting. Spontaneous recovery was also assumed to occur among cases of moderate wasting, where a child with untreated moderate wasting can return directly to the Healthy state. Using the estimated average duration of a severe wasting episode, and all other known rates which collectively determine the prevalence of moderate wasting (either entering or leaving the Moderately Wasted state) the average duration of an episode of moderate wasting was estimated.

\section{Incorporating Seasonality of Incidence: Food insecurity levels, Weather and Diarrheal Disease Trends}

Incorporating Food Insecurity Levels

The factors which influence child wasting are multifaceted. However, it is known that worsening food insecurity is a primary predictor of wasting. Food insecurity directly affects the nutritional status of children by compromising both the quantity and quality of their dietary intake. A direct association between household food insecurity and wasting in developing countries has been previously established, where an increased severity of food insecurity was associated with a higher prevalence of wasting [20]. In Yemen, already facing high levels of food insecurity prior to the conflict, the direct effects of persistent conflict have severely disturbed the country's food distribution mechanisms. As a country which is heavily reliant on imported food, importing about $90 \%$ of its food supply, blockades of the country's main ports have crippled the economy and severely restricted food availability [4]. The decreased availability of food has led to sharp increases in food prices in local markets, and with the majority of Yemenis dependent on market food purchases and widespread unemployment, economic deterioration and a loss of family incomes exacerbating a food crisis, with the result that two-thirds of the Yemeni population do not know where their next meal is coming from [21]. Families must adopt negative coping strategies such as shifting to less preferred or inexpensive food options or limiting their number of meals.

The model aimed to capture the direct relationship between food insecurity levels and wasting, considering how fluctuations in food availability in the country would create variations in incidence rates of wasting among under-five children. The model's incidence transition rates (Healthy to Moderately Wasted, Moderately Wasted to Severely Wasted), representing the probability that a child will develop either moderate or severe wasting, were therefore time-varying and parametric. Monthly minimum food basket prices for the Lahj governorate were used as the primary metric of food insecurity levels. This data came from the World Food Program's (WFP) Monthly Market Watch Reports for Yemen [22]. The minimum food basket in Yemen, endorsed by the country's Food Security and Agriculture cluster, consists of wheat flour, beans, vegetable oil, sugar and salt, considered a household's staple foods. Minimum food basket prices were taken as the primary metric for assessing food insecurity levels for several reasons. First, it has been shown that rising food prices create the primary barrier to food access for most families in Yemen, as the conflict has created widespread unemployment and exacerbated families' financial stress. As food prices have increased, basic food necessities have become out of reach for an increasing number of people, and families cannot secure nutritious food for themselves and their children [21]. The severe depreciation of the local currency caused by the ongoing conflict has led to a considerable rise in the cost of consumer goods since the crisis began, and the continued economic instability has meant that market food prices are often volatile, accounting for the resulting volatility of food insecurity levels since the crisis began. Additionally, variations in the cost of basic food products would also holistically capture reductions in other possible metrics of food insecurity levels - such as food supply, currency depreciation and fuel availability for food transport - and their respective effects on a family's access to food.

In order to establish a baseline for comparison, monthly food basket prices were expressed in relation to pre-crisis food basket prices as reported by the World Food Program, with 2018 and 2019 food basket prices for each month expressed as a percentage of the reported precrisis price. In the data set used, this value ranged between 1.47 and 2.16 during 2018 and 2019. For each of these values, the F/ value was calculated, a value ranging from 0 to 1 , where value of 1 corresponds to the highest relative food insecurity levels. Table 4 a presents the 
corresponding $\mathrm{Fl}$ value range for each food insecurity bracket, and Table $4 \mathrm{~b}$ presents the determined quarterly bracket based on relative food basket prices from September 2018 to October 2019.

\begin{tabular}{|c|c|}
\hline Fl Bracket & Flvalue \\
\hline Bracket 1 & $0.75-1$ \\
\hline Bracket 2 & $0.5-.75$ \\
\hline Bracket 3 & $0.25-0.5$ \\
\hline Bracket 4 & $0-0.25$ \\
\hline
\end{tabular}

Table 4a. Food Insecurity (FI) brackets defined based on $F /$ value.

\begin{tabular}{|c|c|}
\hline Year-Quarter & F1 Bracket \\
\hline $2018-Q 2$ & 4 \\
\hline $2018-Q 3$ & 3 \\
\hline $2018-Q 4$ & 2 \\
\hline $2019-Q 1$ & 1 \\
\hline $2019-Q 2$ & 1 \\
\hline $2019-Q 3$ & 1 \\
\hline $2019-Q 4$ & 2 \\
\hline
\end{tabular}

Table 4b. Food Insecurity Brackets for each quarter from September 2018 to October 2019. Based on relative food basket prices of Lahj.

The effect of food insecurity levels on the incidence rates of both moderate and severe wasting was considered by applying this effect to the duration of an episode of wasting. All estimates of incidence, with incidence defined as the number of new cases which develop over the course of one planning period of length $t$ were based on the following formula:

Equation 1. Incidence $=$ prevalence $\times \frac{t}{\text { duration of episode }}$

Or, expressed in terms of the incidence correction factor $k$ :

$$
\text { Incidence }=\text { prevalence } \times k
$$

This formula was proposed for use in the CMAM model in order to calculate the number of incident cases for a planning period of one year [7]. Based on equation 1, if prevalence is held constant, a longer duration will result in a higher incidence correction factor and thus a higher incidence rate. Based on the established relationship between higher levels of food insecurity and higher rates of incidence of wasting, a simple linear relationship was assumed between the FI value and the duration of an episode, re-calculated on a quarterly basis and referred to as Qduration. This relationship is expressed in Equation 2, where the baseline average duration avgdur, found previously through model calibration, is modulated by the $F / v a l u e$. Using this equation, a higher $F /$ value yields a shorter and thus a higher incidence rate. A delayed effect was assumed, where the effects of a change to food insecurity levels - in the form of a change to incidence - in the current quarter would not be observed until the following quarter. This parameter was considered on a quarterly basis because it was assumed that isolated, monthly food price fluctuations would not create significant disruptions or improvements to overall food security levels and nutritional outcomes; the model sought to capture large-scale and long-term changes that would likely disrupt access to food.

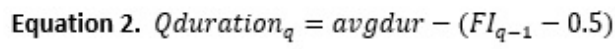

Then, based on the calculated quarterly duration of an episode, the quarterly incidence rate was calculated

using Equation 3. Using the model's estimated prevalence at the end of the previous quarter and an adjusted,

quarterly incidence correction factor $k_{q}$, the incidence rate can be calculated.

Equation 3. Qincidence $_{q}=$ prevalence $_{q-1} \times k_{q} \times .25$

where $k_{q}$ is the quarterly incidence correction factor

$$
k_{q}=\frac{12}{\text { Qduration }_{q}}
$$

Incorporating Weather and Diarrheal Disease Trends

Having established a quarterly incidence rate based on changes to food insecurity levels, next, the effects of diarrheal disease rates were considered. The relationship between diarrheal disease and wasting among children has been well-established - for both moderate and 
severe wasting and diarrhea has been cited as a leading cause of wasting in children under five years old by the World Health Organization $[23,24,25]$. Diarrheal disease has been shown to be a strong predictor of wasting among children because episodes of diarrheal disease inhibit the child's ability to absorb important nutrients necessary for growth. In Yemen, frequent heavy rains and limited sanitation and hygiene services have driven the spread of diarrheal disease [26]. Given the particularly high rates of diarrheal disease among under-five children in Yemen, the model aimed to capture the way in which fluctuations in diarrheal disease rates would directly affect incidence rates of wasting - a relationship that is both well-established in the literature and anecdotally reported by health care workers on the ground in Yemen.

Given that it is known that diarrheal diseases are more likely to spread in period of heavy rains and higher temperatures, we defined a monthly rainfall value ranging from 0 to 0.1 , a weighted sum of historical monthly rainfall and temperatures for Lahj as reported by the World Bank [27]. Relative weightings were determined as those which would most closely match diarrheal disease admission rates from health facilities' clinical register data provided by UNICEF, where it was assumed that a higher rate of admissions corresponded to a higher incidence rate. A comparison of the established monthly rainfall value and diarrheal disease admissions in under-five children is shown in Figure 5, showing the direct association between the two values.

With the quarterly incidence known, the rainfall value would modulate this value to determine a monthly incidence rate. Diarrheal disease was considered on a monthly basis given its more immediate impact on incidence rates of wasting. It was assumed that a change in diarrheal disease rates in the current month would affect the following month's wasting incidence rates. Monthly incidence rates were calculated using Equation 4. The rainfall value was assigned a threshold of 0.04 , where any value below this would not alter the originally calculated incidence rate. This value corresponded to the average rainfall value during the non-rainy season in Lahj.

Equation 4.

Mincidence $_{m}=\left\{\begin{aligned} \text { Qincidence }_{m}+\left(\text { rainfall }_{m-1} \times \text { Qincidence }_{m}\right), & \text { rainfall }_{m-1} \geq 0.04 \\ \text { Qincidence }_{m}, & \text { rainfall }_{m-1}<0.04\end{aligned}\right.$

\section{Results}

Baseline Average Duration of Episode of Moderate and Severe Wasting

After calibrating the model to produce an estimate of the average duration of an episode of severe wasting that would result in the closest match to the 2019 prevalence data as reported by SMART, it was found that an average duration of 4.8 months, for an incidence correction factor $k$ of 2.5. Using this value to estimate the incidence of severe wasting (used to calculate the Moderately Wasted to Severely Wasted transition rate), subsequent model calibration produced an estimate of the average duration of an episode of moderate wasting of 5.3 months, for an incidence correction factor of 2.3. A comparison of the model's outputs and the SMART survey's recorded prevalence using each of these estimates is shown in Figure 6.

\begin{tabular}{|c|c|c|}
\hline & SMART 2019 & Model Output \\
\hline $\begin{array}{c}\text { Severe Wasting } \\
\text { Prevalence }\end{array}$ & 3733 & 3718.7 \\
\hline $\begin{array}{c}\text { Moderate Wasting } \\
\text { Prevalence }\end{array}$ & 21358 & 21350.8 \\
\hline
\end{tabular}

Table 5. Validation of resulting prevalence of moderate and severe wasting in October 2019 using model's adjusted incidence rates.

Monthly Variability in Incidence of Moderate and Severe Wasting

After accounting for food insecurity levels and seasonal diarrheal disease trends, the model produced the monthly incidence rates shown in Figure 7. In order to validate the monthly variations in incidence rates, the estimated monthly incidence rates were validated against treatment admissions data for severe wasting and moderate wasting, respectively, monthly treatment admissions data provided by UNICEF for Lahj. Admissions data was first normalized based on data presenting monthly screening rates in order to ensure that an increase in admissions could be attributed to an increase in incidence and not simply an increase in the number of children screened and subsequently referred to treatment. Upon normalization, the model's estimated incidence rates generally mirrored adjusted admissions rates for both moderate and severe wasting as shown in Figure 7. Table 6 presents validation of the model's resulting prevalence at the end of the model's simulation, during October 2019, after accounting for monthly variability against reported prevalence from the SMART survey.

Page 10/19 
It was found that over the course of the 14 months for which the model was run, the duration of an episode of moderate wasting ranged from 4.7 months to 5.8 months, for an incidence correction factor ranging from 2.0 to 2.6. During this period, the average duration of an episode of severe wasting ranged from 4.1 months to 5.2 months, for an incidence correction factor ranging from 2.3 to 2.9. As expected, both values were lower than the originally assumed value of 7.5 months. Knowing the incidence rate of moderate and severe wasting as well as all other rates which determine the prevalence of each, the model also provided a monthly estimate of the prevalence of both moderate and severe wasting - values generally unknown given that cross-sectional prevalence surveys are conducted only once a year. Additionally, knowing the incidence of both moderate and severe wasting - where it was assumed all severe wasting cases developed from existing moderate wasting cases - it was found that approximately $19 \%$ of children who were moderately wasted developed severe wasting over the course of the model's simulation.

\begin{tabular}{|c|c|c|}
\hline & SMART 2019 & Model Output \\
\hline $\begin{array}{c}\text { Severe Wasting } \\
\text { Prevalence }\end{array}$ & 3733 & 3840.5 \\
\hline $\begin{array}{c}\text { Moderate Wasting } \\
\text { Prevalence }\end{array}$ & 21358 & 21380.9 \\
\hline
\end{tabular}

Table 6. Validation of resulting prevalence of severe and moderate wasting in October 2019 after using model's adjusted incidence rate and accounting for variability.

Using the adjusted incidence rates for moderate and severe wasting, adjusted caseloads were calculated for 2019, with caseload defined as the sum of prevalent cases at the start of the year and incident cases which develop throughout the year. Figures 10 shows a comparison of the original estimated caseload, calculated using an average duration of 7.5 months, and the adjusted caseload for both moderate and severe wasting. As shown in Table 7 , the adjusted moderate wasting caseload was approximately $29 \%$ higher than the originally assumed value, and the adjusted severe wasting caseload was approximately $40 \%$ higher than the originally assumed value.

\begin{tabular}{|l|c|c|}
\hline & Original Estimate & Adjusted Estimate \\
\hline Moderate Wasting Caseload & 55486 & 71950 \\
\hline Severe Wasting Caseload & 13620 & 19080 \\
\hline
\end{tabular}

Table 7. Caseload Comparison before and after adjusting. Comparison of original caseload (using incidence correction factor of 1.6) and adjusted caseloads for moderate and severe wasting.

Using the model's computed estimates of monthly incidence after incorporating the effects of seasonal diarrheal disease trends and food insecurity levels, a monthly estimate of the prevalence of moderate and severe wasting was produced, as shown in Figure 10. Given that cross-sectional prevalence surveys were conducted only once a year in Lahj, monthly estimates of previous of prevalence were previously unknown.

\section{Discussion \& Concusion}

In this manuscript, we provide context-specific estimates of incidence for moderate and severe wasting which consider monthly variability in incidence rates due to changing food insecurity levels and seasonal diarrheal disease trends. From this - and considering all other rates which collectively determine the burden of wasting - we provide monthly estimates of the prevalence of moderate and severe wasting and a framework for holistically assessing the burden of wasting among under-five children. Accurate estimates of the incidence of wasting are critical for projecting the needs of a program and an understanding of how these rates vary, either due to normal seasonal fluctuations and the continued instability caused by the conflict, allows decision-makers to anticipate periods when incidence is expected to increase and plan accordingly.

For both moderate and severe wasting, adjusted incidence rates reached their highest in July, reflecting the peak in treatment admissions for both in July. Given that food insecurity levels for July of 2019 are among the lowest of those in the period examined, this sharp increase is largely the result of the recorded increase in diarrheal disease rates among children in June due to the season's heavy rains. A second peak in the incidence of both moderate and severe wasting occurs around February of 2019. This is likely the result of food insecurity levels reaching the highest recorded levels since the crisis began (with minimum food basket prices were more than double pre-crisis prices) during quarter 4 of 2018. During December 2018, the IPC analysis showed that food insecurity levels in Yemen were at the worst they had 
ever been and warned that the country was on the brink of a full-scale famine [28]. The sharp increase in incidence seen in the following quarter reflects this.

The model's monthly estimates of the prevalence of moderate and severe wasting - after accounting for variability in incidence and allowing the model to run for fourteen simulated months - present several insights about the limitations of basing all planning decisions on an estimate of prevalence. As shown in Figure 10, the prevalence of both moderate and severe wasting varies by season, meaning the month during which a cross-sectional prevalence survey is conducted will affect the estimated caseload for the coming year. Thus, if a SMART survey is conducted in a month during which prevalence is naturally lower, when incidence and subsequently caseloads are calculated using this value, both will be underestimates. While the model's estimated monthly prevalence for moderate and severe wasting does, expectedly, reflect the same general trends as the computed monthly incidence, even with prevalence data recorded at more frequent intervals, it is difficult to draw firm conclusions from prevalence alone.

While monthly prevalence does follow the same general trends as those found for incidence, fluctuations among prevalence are much more subtle, with prevalence for both moderate and severe wasting remaining relatively stable throughout the year. For example, the steep increase in the incidence of both moderate and severe wasting around January of 2019 is reflected within prevalence estimates with a much less extreme increase. It is difficult to conclude that an increase in incidence must be matched with an increase in prevalence as prevalence is affected by several factors. If the incidence of both moderate and severe wasting increases, while we may expect to see a higher prevalence of moderate wasting, this also means more cases of moderate wasting are progressing to severe wasting. Additionally, because the model assumes that during any given month, the probability of mortality among children with severe wasting or moderate wasting, respectively, remains constant, then when there is a greater number of children who are wasted, mortality due to wasting is expected to be higher, also affecting the overall prevalence of both. While the initial approach in which prevalence data was collected only once per year presented major limitations in that it was unknown whether new cases were developing within the span of this period, the model's results show that even more regular collection of prevalence data presents limitations; without an accurate estimation of incidence, it is difficult to derive insights from prevalence data which can guide planning decisions. Estimates of monthly incidence - presenting the number of children developing new cases of moderate and severe wasting will therefore require treatment - provide insights that are of practical use to decision-makers.

The model's computed incidence rates align with the consensus within the literature that a single incidence correction factor of 1.6 is insufficient and results in underestimates of caseload. As shown in Table 7, previous estimates did in fact lead to considerable underestimates of caseload, leaving populations of children in need of treatment unaccounted for. Because of this, when target caseloads are calculated to plan for the coming year, relying on the original estimate to guide the planning of resources and services may lead to potential shortages. An understanding of when incidence rates are expected to increase during the year also allows decision-makers to anticipate such increases and target their efforts during periods when the burden of wasting is expected to worsen. Aside from normal, seasonal fluctuations, a consideration of underlying factors such as food insecurity which directly influence rates of wasting and which are highly variable as a result of the conflict allows for estimates reflecting the instability of a crisis setting like Yemen.

To our knowledge, while several cohort studies have sought to adjust incidence estimates for moderate wasting. Several studies, using data from cohort studies in several African nations, have found context-specific incidence correction factors [18, 9,19]. All found the estimated duration of 7.5 months resulted in underestimates of caseload. However, as the authors of these works note, these results are not intended to be generalized to other regions where the context, treatment coverage, food availability, and disease prevalence are likely very different. Thus, our results cannot be compared to those found in other contexts. They do, however, confirm the assumption that incidence correction factors vary considerably by context, and a single estimate cannot be appropriate. While it is known that seasonal variations will affect incidence rates of wasting, possible approaches for accounting for seasonal variability have not been extensively explored [29]. Each of the aforementioned studies exploring context-specific incidence correction factors, assumes a constant rate of incidence, though recognizing the limitations of doing so.

Other works have suggested that a general approach to accounting for seasonal trends in rates of wasting could involve examining the country's seasonal agricultural calendar, where typical agricultural production patterns determine the year's food insecurity trends. In doing so, one could determine at what point during the year the "lean seasons" fall - the period between planting and harvesting when job opportunities are scarce and food stocks dwindle - and map these patterns onto rates of wasting [30, 31]. In the context of Yemen, however, this likely provides little insight. As a country with limited agricultural productivity that is now almost entirely reliant on commercial imports of food, an agricultural calendar would likely have little bearing on the burden of wasting. Additionally, the situation in a conflict-affected setting like Yemen is inherently unstable; the availability and accessibility of food does not follow a regular, predictable pattern as it is largely dependent on developments within the conflict. Our approach seeks to account for this by taking inputs that reflect the ever-evolving nature of the context.

Page 12/19 
As is the case with many mathematical models seeking to capture complex processes, our model had several limitations. First, a fundamental property of Markov models is that of "memorylessness" - the assumption that the future states depend only on the current state and not any past states [10]. Though it is known, for example, that a child who spontaneously recovers from moderate wasting is at a greater risk of developing moderate wasting again, the model cannot consider this as the child would return to the Healthy state in its simulation, and would therefore have the same probability of becoming moderately wasted as a child who had never previously been moderately wasted. Another case where this may present a limitation is when children may default from treatment and return to a malnourished state and therefore be considered the same as someone who had never before been in treatment. However, given that we did not aim to examine these cases at such an individual level and that rates of default and spontaneous recovery were relatively low, the effects of these limitations on our results were likely minimal.

Though we sought to capture variability in incidence estimates, the assumption that other rates would remain constant presented several limitations. It is likely that mortality rates vary seasonally; however, given that it would be unethical to study a cohort of untreated children while denying them treatment, little is known about outcomes of untreated wasting. Existing estimates of untreated case fatality rates for moderate and severe wasting were used for this model, but given that this data is limited, variations in these rates could not be explored. Among the most uncertain of the model's rates is the spontaneous recovery rate for moderate and severe wasting. Because spontaneous recovery tends to happen by chance, and with the changing situation in Yemen, there is little data representing the current context. Cohort studies tracking untreated cases of wasting - which would monitor rates of spontaneous recovery - are scarce given the ethical limitations of doing so; those available were conducted before the onset of CMAM. Given this, data from other contexts was used to estimate these rates, which posed several limitations given that spontaneous recovery happens by chance and is dependent on many contextual factors. Because of this, our model's spontaneous recovery rates were uncertain and may have affected the resulting estimates of incidence.

Several limitations were presented by the limited data available for validation. Given that we sought to estimate monthly incidence rates and that prevalence data in Yemen is collected only on an annual basis, treatment admissions data was used to validate the monthly trends found in the computed incidence rates for moderate and severe wasting. While admissions rates were adjusted for screening - to ensure that an increase in admissions was not merely the result of an increase in screening - this approach presented several limitations. With screening accounted for, it was assumed that all other variations in admissions rates were the result of variations in incidence and thus the general trends they presented were expected to be the same. However, aside from screening, other factors such as socioeconomic status may hinder someone's ability to access treatment. Because of this, we did not attempt to establish or assess rigid correlations between treatment admissions and our estimated monthly incidence; admissions were taken as a proxy for incidence but were only examined for overall, general trends. An additional limitation presented in validating our model's results against SMART survey results; this assumes that the SMART survey results are in fact accurate. However, because SMART relies on representative sampling in order to estimate prevalence for the entire governorate, it is expected that there be some uncertainty in its results. Additionally, because SMART surveys in Yemen are conducted annually, the absence of intermediate data points meant that monthly prevalence could not be validated. Given that conducting widescale cross-sectional prevalence surveys is costly and humanitarian agencies' face increasingly limited funding, it is expected that this data will be scarce. Despite this, the model makes use of the available data to offer new insights, improve upon the existing approach for estimating wasting and strengthen understandings of the burden of wasting in Yemen. In general, data coming from the region is limited and information systems weak due to the conflict; in order to provide any new and practical insights beyond those available from the limited data in a conflict zone, that data cannot be dismissed due to its limitations but its limitations may be addressed and this data utilized to improve upon what is available.

While it was established that relative food basket prices would serve as a holistic metric for food insecurity metrics, doing so was also not without its limitations. The factors which determine food insecurity are multifaceted and difficult to concretely define. For example, it is known that the World Food Program distributes food assistance to families at risk of and facing food insecurity, which likely plays a role in alleviating the burden of wasting. Additionally, in addition to treatment services provided by various humanitarian actors on the ground in Yemen, the World Food Program provides preventative programs including Blanket Supplementary Feeding Programs (BSFPs) and various community outreach programs [6]. While each of these interventions likely plays a role in alleviating the burden of wasting and their incorporation into the model was considered, little work has been done to quantify their relative impacts making it difficult to incorporate them into the model framework.

Despite its limitations, our model can provide decision-makers with important insights about the expected burden of wasting. In addition to providing more accurate estimates of caseload and presenting seasonal variations in incidence, our model can also be used as a predictive tool for health workers on the ground. By considering inputs that reflect changing conditions on the ground as a result of many of the conflict's indirect effects, decision-makers can utilize the model's framework to adjust its parameters and understand how the burden of wasting is expected to change with changing conditions and plan accordingly. Additionally, rather than considering the development of

Page 13/19 
moderate wasting, severe wasting and severe wasting with complications independently of each other, the model framework allows for a consideration of the complete system of interactions that they form. With more accurate estimates of the incidence of severe wasting, the model captures the rate at which moderate wasting is expected to develop into severe wasting. By representing the burden of wasting in a model of this form, the model can not only produce expected estimates, but also be used as a tool to simulate various scenarios in order to guide planning decisions. For example, by adjusting the rate of treatment for moderate wasting and running the model for several months, decision-makers can understand the long-term effects of doing so on not just the burden of moderate wasting, but also the burden of severe wasting and severe wasting with complications in the long-term. Thus, accurate estimates of incidence are not only critical in determining the number of children in need, but also in more holistically assessing the nutrition situation.

In humanitarian settings such as Yemen where funding and resources are extremely limited, these understandings can help ensure that limited resources are allocated most effectively. Additionally, the model's ability to holistically capture all determinants of the monthly prevalence of wasting may provide a potential alternative to conducting in-person cross-sectional surveys such as SMART, allowing humanitarian agencies to direct efforts and funds elsewhere. Future work may entail extending the model framework to other conflicteffected settings in order to produce more accurate caseload estimates and consider the expected instability of conflict settings. Doing so may validate the utility and generalizability of the model and in other contexts. The model may allow healthcare workers to allocate already limited resources and funding most effectively, plan for anticipated changes in the burden of wasting and provide children with the nutritional support they require.

\section{Abbreviations}

CMAM: Community-based Management of Acute Malnutrition

MAM: Moderate Acute Malnutrition

SAM: Severe Acute Malnutrition

SMART: Standardized Monitoring and Assessment of Relief and Transitions

OTP: Outpatient Therapeutic Feeding Program

TFC: Therapeutic Feeding Center

TSFP: Targeted Supplementary Feeding Program

IPC: Integrated Food Security Phase Classification

\section{Declarations}

\section{Ethical Approval and Consent to Participate}

All human based data was deidentified and shared by UNICEF following ethical guidelines.

\section{Consent for Publication}

Not applicable

\section{Availability of data and materials}

The data that support the findings of this study are available from UNICEF but restrictions apply to the availability of these data, which were used under license for the current study, and so are not publicly available. Data are however available from the authors upon reasonable request and with permission of UNICEF.

\section{Competing Interests}

The authors state that the institutions of Boston University (BU) and the United Nations International Children's Fund (UNICEF) are currently involved in a financial partnership.

Funding 
The completion of this study was made possible with funding provided by UNICEF. This research was also supported by the Undergraduate Research Opportunities Program at Boston University.

\section{Authors' Contributions}

RAH was a main contributor to the data analysis and interpretation, designed the model and was a main contributor in writing the manuscript. MHZ provided major guidance in the development of the model and the creation of the manuscript. MPS assisted in model development and data analysis. SG and ER provided technical support and nutritional expertise which informed the model's development. NAD compiled and provided data, assisted in developing the model and provided guidance in data interpretation and insight on ground realities.

FS and MKD provided guidance in the study.

MHZ, NAD, MPS, SG, and ER all provided edits to the manuscript. All authors read and approved the final manuscript.

\section{Acknowledgements}

Not applicable

\section{Authors' Information}

NAD, FS and MKD have all worked on the ground in Yemen and are familiar with the country's current realities.

\section{References}

1. Brück, T., \& D'errico, M. (2019). Food security and violent conflict: Introduction to the special issue. World Development, 117, $167-171$. doi:10.1016/j.worlddev.2019.01.007

2. Martins, V. J., Toledo Florêncio, T. M., Grillo, L. P., do Carmo P Franco, M., Martins, P. A., Clemente, A. P., Santos, C. D., de Fatima A Vieira, M., \& Sawaya, A. L. (2011). Long-lasting effects of undernutrition. International journal of environmental research and public health, 8(6), 1817-1846. https://doi.org/10.3390/ijerph8061817

3. Loewenberg S. (2015). Conflicts worsen global hunger crisis. Lancet (London, England), 386(10005), $1719-1721$. https://doi.org/10.1016/S0140-6736(15)00734-5

4. Dureab, F., Al-Falahi, E., Ismail, O., Al-Marhali, L., Al Jawaldeh, A., Nuri, N. N., Safary, E., \& Jahn, A. (2019). An Overview on Acute Malnutrition and Food Insecurity among Children during the Conflict in Yemen. Children (Basel, Switzerland), 6(6), 77. https://doi.org/10.3390/children6060077

5. Yemeni children suffer record rates of acute malnutrition, putting 'entire generation' at risk | | UN News. (n.d.). Retrieved November 09, 2020, from https://news.un.org/en/story/2020/10/1076272

6. Guidelines for Community-Based Management of (Severe \& Moderate) Acute Malnutrition. Retrieved from https://www.humanitarianresponse.info/sites/www.humanitarianresponse.info/files/documents/files/final_yemen_cmam_guidelines__feb_2014.pdf

7. Myatt, M. (2012). How do we estimate case load for SAM and/or MAM in children 6-59 months in a given time period. Llawryglyn: Brixton Health. https://doi.org/10.3390/children6060077

8. Garenne, M., Willie, D., Maire, B., Fontaine, O., Eeckels, R., Briend, A., \& Van den Broeck, J. (2009). Incidence and duration of severe wasting in two African populations. Public health nutrition, 12(11), 1974-1982. https://doi.org/10.1017/S1368980009004972

9. Sheila Isanaka, Ellen O'Neal Boundy, Rebecca F. Grais, Mark Myatt, André Briend, Improving Estimates of Numbers of Children With Severe Acute Malnutrition Using Cohort and Survey Data, American Journal of Epidemiology, Volume 184, Issue 12, 15 December 2016, Pages 861-869, https://doi.org/10.1093/aje/kww129

10. Sonnenberg FA, Beck JR. Markov models in medical decision making: a practical guide. Med Decis Making. 1993 Oct-Dec;13(4):322-38. doi: 10.1177/0272989X9301300409. PMID: 8246705.

11. 2019 SMART Survey Lahj, Yemen. Retrieved from https://www.humanitarianresponse.info/en/operations/yemen/nutrition

12. 2018 SMART Survey Lahj, Yemen. Retrieved from: https://www.humanitarianresponse.info/en/operations/yemen/nutrition

13. El Bcheraoui, C., Jumaan, A. O., Collison, M. L., Daoud, F., \& Mokdad, A. H. (2018). Health in Yemen: losing ground in war time. Globalization and health, 14(1), 42. doi:10.1186/s12992-018-0354-9

Page 15/19 
14. Javan, R., Kooshki, A., Afzalaghaee, M., Aldaghi, M., \& Yousefi, M. (2017). Effectiveness of supplementary blended flour based on chickpea and cereals for the treatment of infants with moderate acute malnutrition in Iran: A randomized clinical trial. Electronic physician, 9(12), 6078-6086. https://doi.org/10.19082/6078

15. Sachdev, H. S., Sinha, S., Sareen, N., Pandey, R. M., \& Kapil, U. (2017). Survival and Recovery in Severely Wasted Under-five Children Without Community Management of Acute Malnutrition Programme. Indian pediatrics, 54(10), 817-824. https://doi.org/10.1007/s13312-017-1142-y

16. Olofin, I., McDonald, C. M., Ezzati, M., Flaxman, S., Black, R. E., Fawzi, W. W., Caulfield, L. E., Danaei, G., \& Nutrition Impact Model Study (anthropometry cohort pooling) (2013). Associations of suboptimal growth with all-cause and cause-specific mortality in children under five years: a pooled analysis of ten prospective studies. PloS one, 8(5), e64636. https://doi.org/10.1371/journal.pone.0064636

17. Barba, F. M., Huybregts, L., \& Leroy, J. L. (2020). Incidence Correction Factors for Moderate and Severe Acute Child Malnutrition From Two Longitudinal Cohorts in Mali and Burkina Faso. American Journal of Epidemiology. https://doi.org/10.1093/aje/kwaa139

18. Bulti, A., Briend, A., Dale, N. M., De Wagt, A., Chiwile, F., Chitekwe, S., Isokpunwu, C., \& Myatt, M. (2017). Improving estimates of the burden of severe acute malnutrition and predictions of caseload for programs treating severe acute malnutrition: experiences from Nigeria. Archives of public health = Archives belges de sante publique, 75, 66. https://doi.org/10.1186/s13690-017-0234-4

19. Sheila Isanaka, Rebecca F. Grais, André Briend, Francesco Checchi, Estimates of the Duration of Untreated Acute Malnutrition in Children From Niger, American Journal of Epidemiology, Volume 173, Issue 8, 15 April 2011, Pages 932940, https://doi.org/10.1093/aje/kwq436

20. Singh A, Singh A, Ram F. Household food insecurity and nutritional status of children and women in Nepal. Food Nutr Bull. 2014 Mar;35(1):3-11. doi: 10.1177/156482651403500101. PMID: 24791573.

21. Missiles and Food: Yemen's Man-Made Food Security Crisis (Issue brief). (n.d.). OXFAM.

22. Yemen Monthly Market Watch. Retrieved from https://www.wfp.org/publications/yemen-monthly-market-watch-2019

23. Kenneth H. Brown, Diarrhea and Malnutrition, The Journal of Nutrition, Volume 133, Issue 1, January 2003, Pages $328 \mathrm{~S}-$ 332S, https://doi.org/10.1093/jn/133.1.328S

24. Yip R, Sharp TW. Acute Malnutrition and High Childhood Mortality Related to Diarrhea: Lessons From the 1991 Kurdish Refugee Crisis. 1993;270(5):587-590. doi:10.1001/jama.1993.03510050053026

25. Sepulveda J, Willett W, Munoz A (1988) Malnutrition and diarrhea. A longitudinal study among urban Mexican children. Am J Epidemiol 127(2): 365- 76.

26. (2019, March). Two years since world's largest outbreak of Acute Watery Diarrhea and Cholera, Yemen witnessing another sharp increase in reported cases with number of deaths continuing to increase. https://www.unicef.org/press-releases/two-years-worldslargest-outbreak-acute-watery-diarrhea-and-cholera-yemen-witnessing

27. World Bank. Yemen Historical Climate Data. Retrieved from: https://climateknowledgeportal.worldbank.org/country/yemen/climatedata-historical

28. Yemen: Acute Food Insecurity Analysis. Retrieved from: https://reliefweb.int/report/yemen/yemen-ipc-acute-food-insecurity-analysisdecember-2018-january-2019\#: :text=According\%20to\%20the\%20latest\%20IPC,IPC\%20Phase\%203\%20(Crisis).

29. Egata, G., Berhane, Y., \& Worku, A. (2013). Seasonal variation in the prevalence of acute undernutrition among children under five years of age in east rural Ethiopia: a longitudinal study. BMC public health, 13, 864. https://doi.org/10.1186/1471-2458-13-864

30. Arsenault, J. E., Nikiema, L., Allemand, P., Ayassou, K. A., Lanou, H., Moursi, M., De Moura, F. F., \& Martin-Prevel, Y. (2014). Seasonal differences in food and nutrient intakes among young children and their mothers in rural Burkina Faso. Journal of nutritional science, 3 , e55. https://doi.org/10.1017/jns.2014.53

31. Ravaoarisoa, L., Rakotonirina, J., Randriamanantsaina, L. et al.Food consumption and undernutrition variations among mothers during the post-harvest and lean seasons in Amoron'i Mania Region, Madagascar. BMC Public Health 19, 1005 (2019). https://doi.org/10.1186/s12889-019-7333-9

\section{Figures}




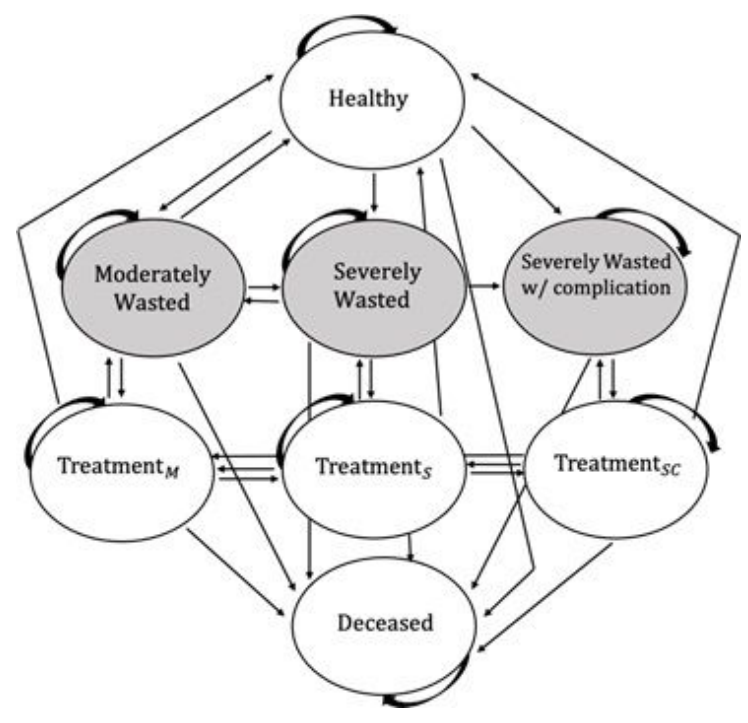

Figure 1

Model Framework. Arrows depict all possible transitions between nutrition states. Gray states indicate a state where a child is wasted but not currently in treatment. Treatment $(\mathrm{M}$,$) Treatment (\mathrm{S}$,$) and Treatment (SC) refer to treatment for moderate wasting, severe wasting, and$ severe wasting with complications, respectively.

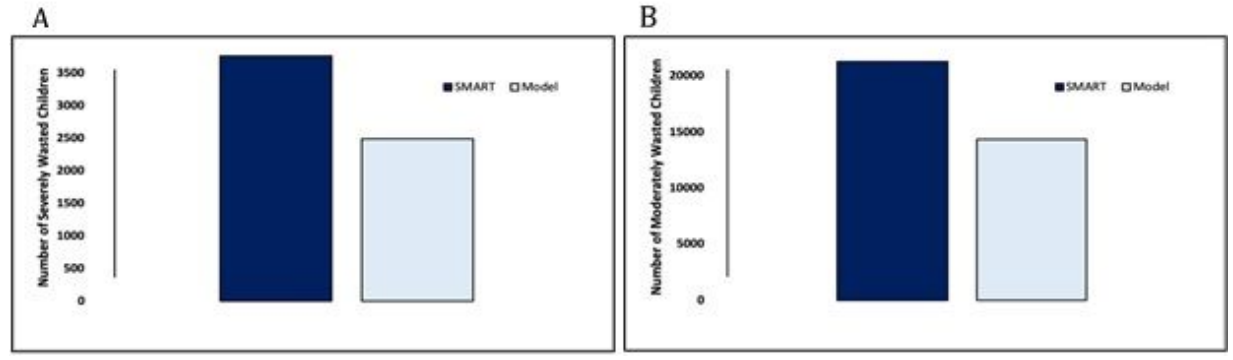

\section{Figure 2}

Prevalence of moderate wasting (A) and severe wasting (B). Comparison of prevalence produced by model compared at end of October 2019 from Lahj SMART survey and prevalence produced by model using the estimated 7.5-month duration of episode when run until October 2019.

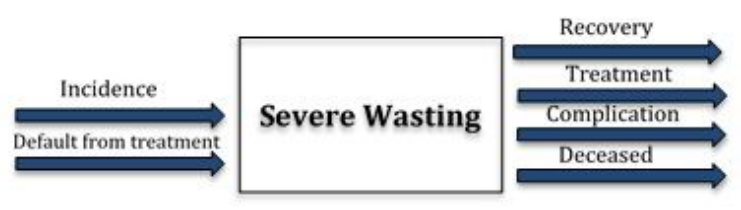

\section{Figure 3}

Magnified view of rates determining severe wasting prevalence within model framework

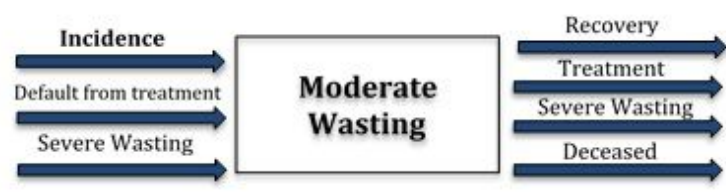

\section{Figure 4}

Magnified view of rates determining moderate wasting prevalence within model framework. 


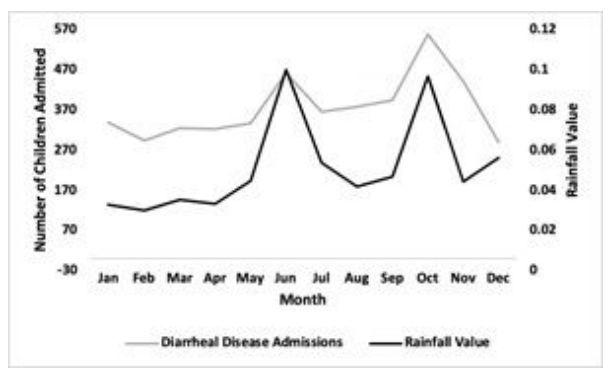

\section{Figure 5}

Seasonality of Diarrheal Disease in Lahj. Diarrheal disease admissions compared against established rainfall value.

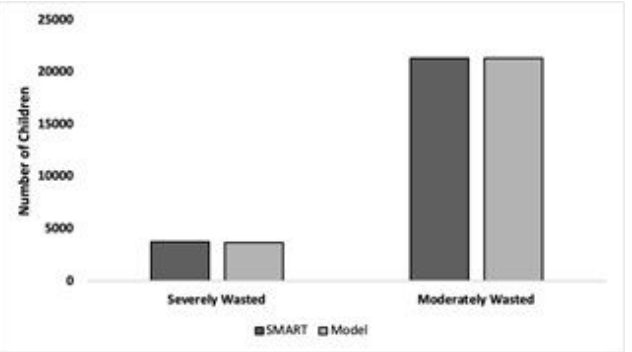

\section{Figure 6}

Validation of Prevalence after Adjusting Incidence. Prevalence of severe and moderate in October 2019 produced by model using adjusted incidence rates compared to SMART survey results.
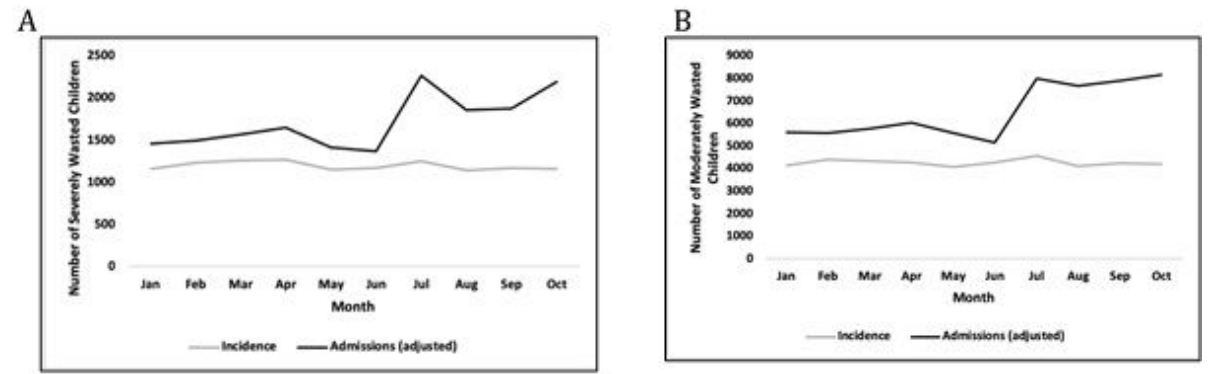

\section{Figure 7}

Monthly Incidence of Severe Wasting (A) and Moderate Wasting (B) September 2018 to October 2019.

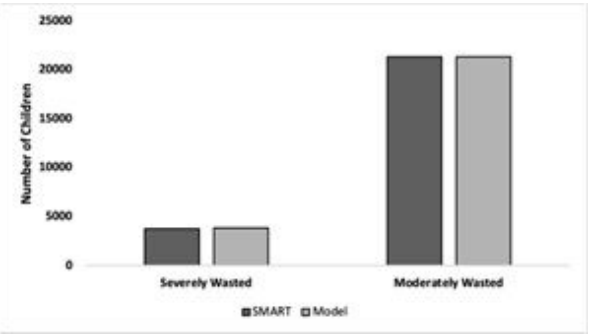

\section{Figure 8}

Prevalence of severe and moderate wasting in October 2019 produced by model using adjusted incidence rates and after accounting for variability compared to SMART survey results. 

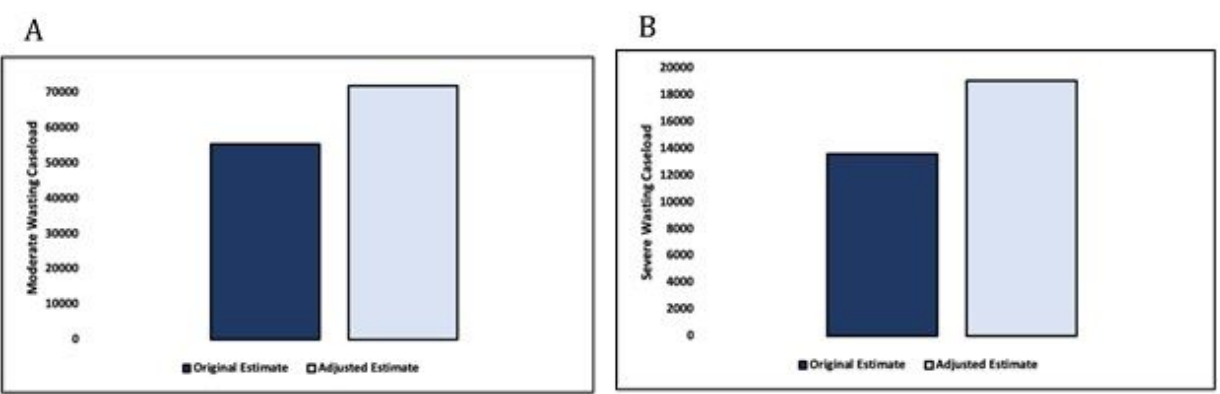

\section{Figure 9}

Comparison of original and adjusted moderate wasting (A) and severe wasting (B) caseload. Original estimate refers to the value produced using an incidence correction factor of 1.6 while adjusted estimate refers to the estimate produced by the model after adjusting the incidence correction factor and accounting for food insecurity and diarrheal disease trends.


\section{Figure 10}

Monthly Prevalence of severe wasting (A) and moderate wasting (B) from model simulation of September 2018 to October 2019 after applying food insecurity and seasonal disease trends 